\title{
Obstetric Versus Traumatic Vulvar Hematoma: Two Case Report and Review
}

\author{
Carmen E. Badillo-Bercebal ${ }^{1}$, Sonia De-Miguel-Manso', ${ }^{1,2}$, Victoria Pascual-Escudero', Cristina Álvarez-Colomo ${ }^{1,2}$, Dakota Viruega- \\ Cuaresma1, Elena García-García ${ }^{1}$. \\ ${ }^{1}$ Servicio de Obstetricia y Ginecología, Hospital Clínico Universitario de Valladolid, Gerencia Regional de Salud de Castilla y León (SACYL),
} España.

2Departamento de Pediatría e Inmunología, Obstetricia y Ginecología, Nutrición y Bromatología, Psiquiatría e Historia de la Ciencia, Facultad de Medicina, Universidad de Valladolid, España.

*Corresponding author: Carmen E. Badillo-Bercebal, Servicio de Obstetricia y Ginecología, Hospital Clínico Universitario de Valladolid, Gerencia Regional de Salud de Castilla y León (SACYL), España.

Received date: April 14, 2021; Accepted date: April 20, 2021; Published date: May 15, 2021

Citation: Carmen E. Badillo-Bercebal, Sonia D. Manso, Victoria P. Escudero, Cristina Á. Colomo, Dakota Viruega-Cuaresma, Elena G. García1. (2021) Obstetric Versus Traumatic Vulvar Hematoma: Two Case Report and Review. J. Obstetrics Gynecology and Reproductive Sciences 5(4); DOI: $10.31579 / 2578-8965 / 069$

Copyright: (C) 2021, Carmen E. Badillo-Bercebal, This is an open access article distributed under the Creative Commons Attribution License, which permits unrestricted use, distribution, and reproduction in any medium, provided the original work is properly cited.

\section{Abstract}

Background: Vulvar hematomas are more frequent in the obstetric population generally as a consequence of episiotomies, precipitated or instrumental deliveries. The most common causes in the general population are accidental trauma or spontaneous rupture of blood vessels. Management in both etiologies depends on size, involvement of adjacent organs, hemodynamic compromise, and the existence of fractures or lacerations that need to be repaired. Edema is a common complication usually resolved with conservative management.

Clinical presentation: We present two cases. A traumatic vulvar hematoma in a 22 -year-old patient and an obstetric vulvar hematoma in a 30-year-old primigravida which already presented pitting edema prior to delivery. Both cases required surgical drainage due to clinical evolution and poor pain control.

Conclusions: The approach can be conservative or surgical depending on several factors but the use of intravenous analgesia is essential for pain management. Although hematomas related with traumatic causes are less frequent than puerperal ones, they can be very dangerous if they are associated with arterial bleeding that leads to hemodynamic instability.

Key words: vulvar hematoma, vulvar edema, surgical drainage

\section{Introduction}

A hematoma is defined as a spontaneous, traumatic or postsurgical collection of blood in soft tissues or surgical sites [1].

The most external components of the vulva (mons pubis and labia majora) share a very vascularized profuse adipose tissue, which performs a protective function in adults. In girls, these fat pads under the labia majora have not yet developed and therefore, vulvar trauma in childhood is more frequent [2].

The genitalia's vascularization increases during pregnancy, which explains why vulvar hematomas are much more frequent in the obstetric population compared to the general population, where they only represent $0.8 \%$ of all gynecological emergencies [3]. The incidence of puerperal vulvar hematomas is 1-2 per 1000 deliveries [4], generally as a consequence of episiotomies, precipitated or instrumental deliveries [5]. The most common cause of non-obstetric vulvar hematomas is accidental trauma; although we must not lose sight of other causes such as sexual abuse or spontaneous rupture of blood vessels as a consequence of aneurysms, varicose veins or arteriovenous malformations [3].

Morbidity and treatment differ substantially depending on the extent and etiology. Obstetric hematomas rarely lead to hemodynamic instability and therefore their management is mainly conservative (5); on the other hand, post-traumatic hematomas are more frequently related to arterial vessel rupture, an exponential increase of size and pain, and the need for surgical drainage [3].

A second challenge in the approach to this pathology is the edema that is frequently associated [6]. Mild or moderate vulvar edema can occur in pregnant or non-pregnant women associated with multiple causes: trauma, infection, inflammation or neoplasia [7]. Generally, for mild and moderate cases, conservative management focused on symptomatic relief is enough [8]. In severe or refractory cases, treatment with diuretics and even the administration of intravenous albumin is necessary; reserving mechanical drainage for highly selected cases in which medical treatment fails [9]. 
We present two cases of vulvar hematomas with very different etiologies that, nevertheless, required a similar approach.

\section{Clinical Presentation}

\section{CASE 1}

22-year-old woman with no allergies or medical-surgical history of interest, nullipara, menarche at 13 years of age and regular cycles. She presented to the emergency room with intense genital pain after a bicycle accident, reporting disabling inflammation of the area that made urination and sitting impossible and that had increased considerably in size in the last 3 hours. She was referred to the gynecology emergency room because the pain could not be controlled with oral or intravenous analgesia. Examination revealed a collection of the right labia majora, with a purplish color and a surface under tension, approximately $12 \mathrm{~cm}$ in length and $6 \mathrm{~cm}$ in width, very painful on palpation, suggesting a vulvar hematoma. The urethra and other vulvar structures were displaced by the described collection. It was accompanied by edema in the right lower lip. [FIGURE 1]

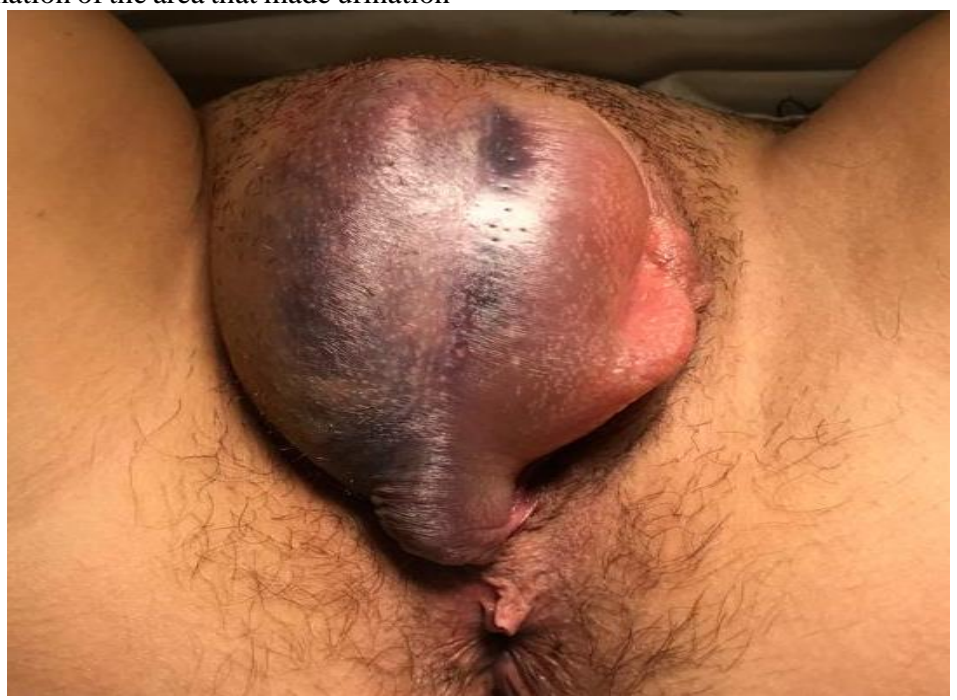

Figure 1. Appearance of the vulvar collection upon admission that suggested hematoma with occlusion of the urethral meatus and other vulvar structures.

A first drainage was performed surgically with a $2-3 \mathrm{~cm}$ incision at the point of maximum fluctuation of the right labia majora, cleansing and mechanical debridement of the organized hematoma, we placed a Penrose-type drain and permanent bladder catheterization. After emptying plenteous clots, no active bleeding point was observed. In addition, oral treatment was started with prophylactic antibiotics, streptokinase, and oral prednisone. In the early postoperative period, a clinical worsening was evidenced, with an increase of the size of the hematoma that distorted the vulvar anatomy and blisters on the labia minora [FIGURE 2]. A surgical revision was performed 19 hours after the first surgery, widening the initial incision. Venous bleeding point was observed from the right bulbocavernosus muscle, haemostasis was carried out by layered closure with loose Vicryl suture; and 2 drains were placed in a slope position.

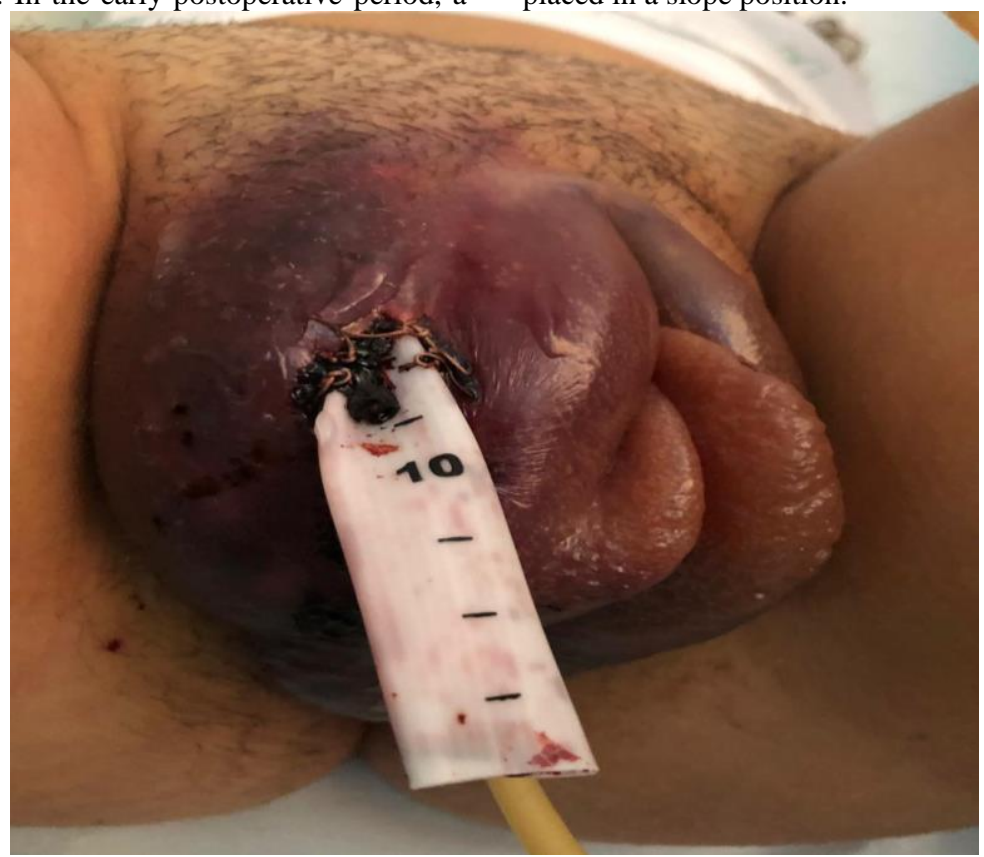


Clinical improvement during the postoperative evolution was remarkable, controlling pain with oral analgesia, and restarting the ambulation and spontaneous urination. The drains were removed after a week and she was discharged with persistent moderate soft vulvar edema, and maintaining treatment with corticosteroids and streptokinase for further 7 days. Postoperative check-up was carried out one month later, observing a scar in good condition, complete resolution of the edema and the absence of sequelae, with resumption of normal physical and sexual activity [FIGURE 3].

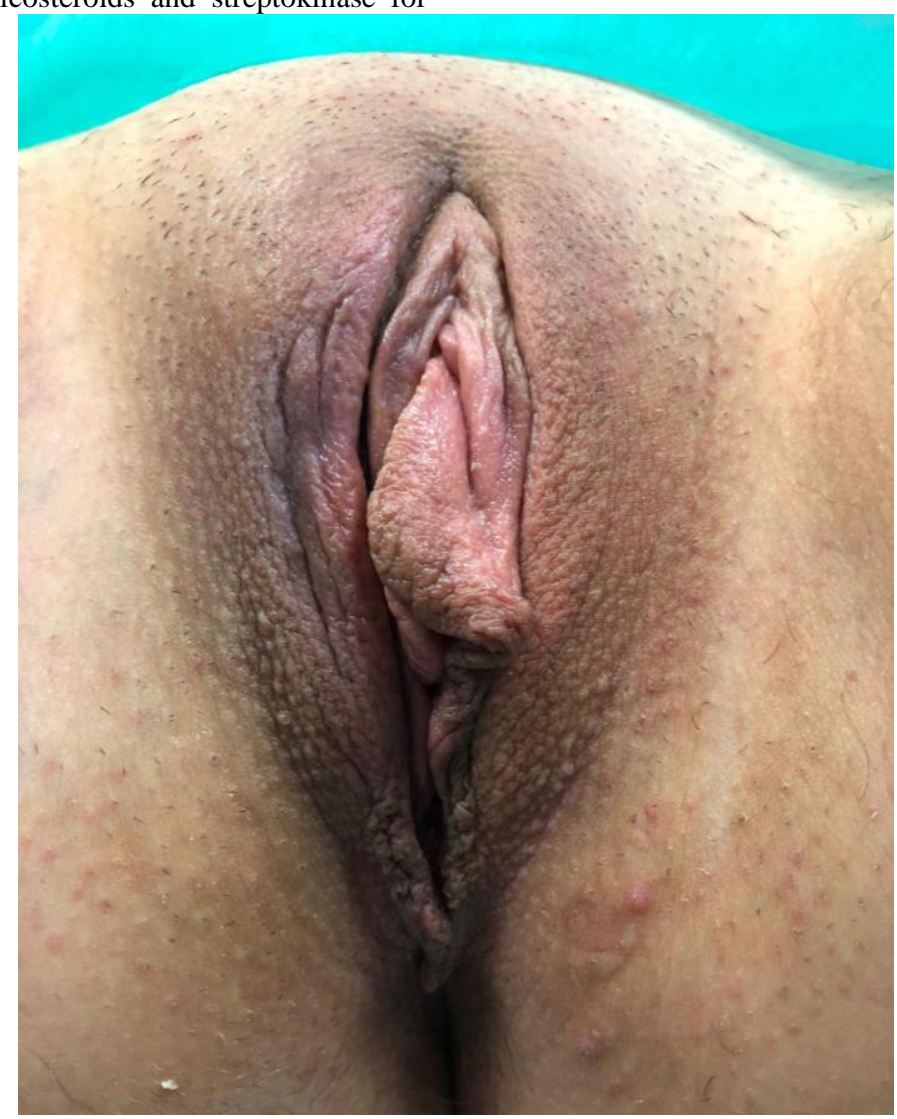

Figure 3. Complete resolution of the edema and the absence of sequelae one month later.

\section{CASE 2}

30-year-old primigravida, with no allergies or relevant surgical history. Diagnosed with Type 1 Diabetes Mellitus at age 19 and on treatment with gliclazide since then. As a complication, she developed diabetic retinopathy that required intravitreal injections of Ranibizumab every 28 days. Pregnancy control was carried out in the high-risk obstetric unit, requiring 16 units of insulin Levemir® (long-acting insulin analog) daily (11-0-5).

Labor was induced at 39 weeks and 5 days due to suboptimal glycemic control. Prior to starting induction, pitting edema up to the knees, slight edema in hands and vulva were observed [for which compression stockings were prescribed]. Induction was started with vaginal prostaglandins, and continued with oxytocic stimulation under effective epidural analgesia. She reached full dilation after 24 hours of cervical ripening and 6 hours of active labor. It was necessary a vacuum extraction due to a non-reassuring cardiotocography, with moderate difficulty in extracting the posterior shoulder because of an assimilated left hand. Upon inspection of the birth canal, a second-degree perineal tear was observed that encompassed the vagina and the root of right labia minora, which was sutured by layered closure.

During the early puerperium, she began with intense pain in genital area that difficult mobility in decubitus and standing. Physical examination revealed severe generalized vulvar edema, which had worsened compared to her prepartum condition. In the absence of improvement with intravenous analgesia, local ice and Glucosmón ${ }^{\circledR}$ (33\% hypertonic glucose solution), she was explored again, observing an enlarged, swollen, violaceous right labia majora, with a very painful tension zone in the lower third, suggestive of a hematoma, which extended along the entire labia and the introitus [FIGURE 4]. 10 hours after delivery, we proposed surgical revision and drainage under anesthesia and placement of a permanent bladder catheter. The main surgical finding was a vulvar hematoma that dissected the subcutaneous plane along the entire right labia majora, with an area of maximum tension on the inner side at the introitus level. It was accompanied by a large edema that encompassed bilateral labia majora and introitus declining area. A mechanical debridement and hemostasis was made [FIGURE 5], and a Penrose-type drain was placed. 


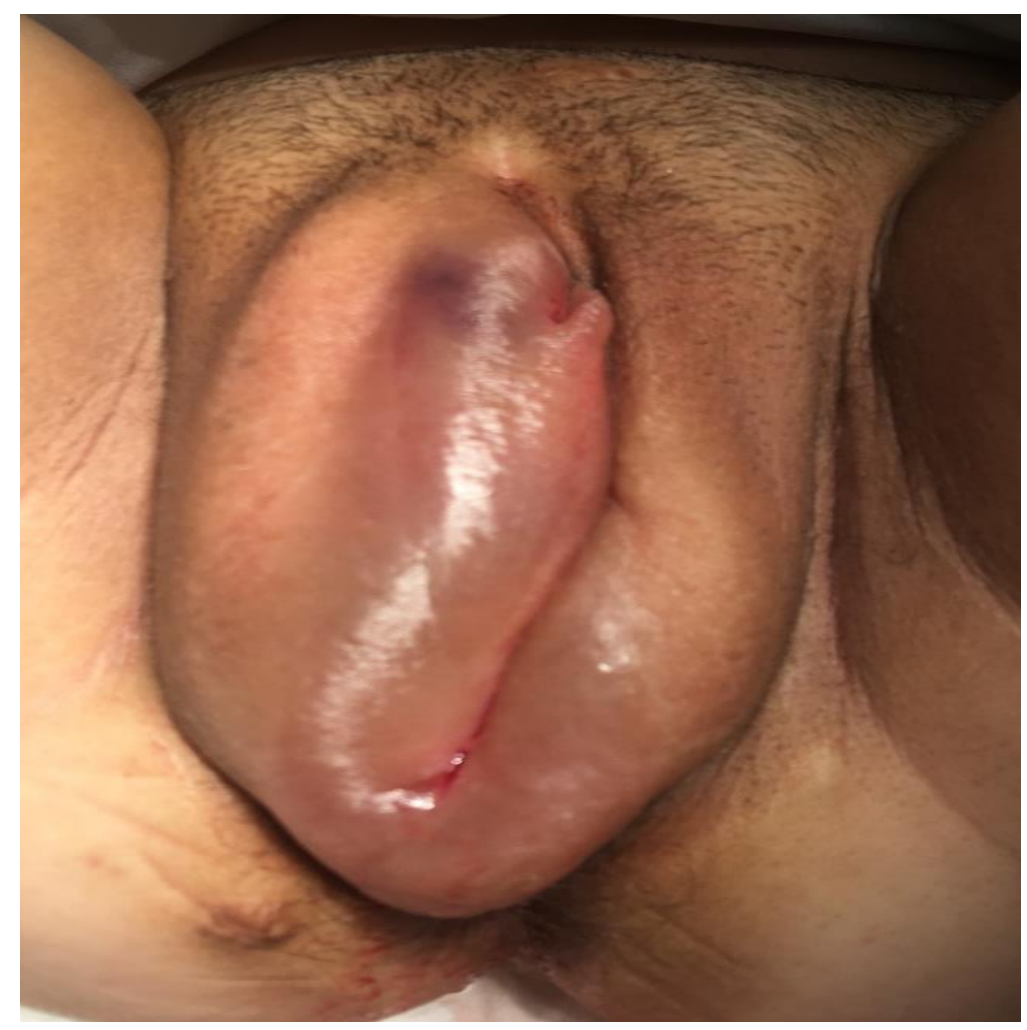

Figure 4. Generalized vulvar edema with suspected hematoma in the lower third of the right labia majora.

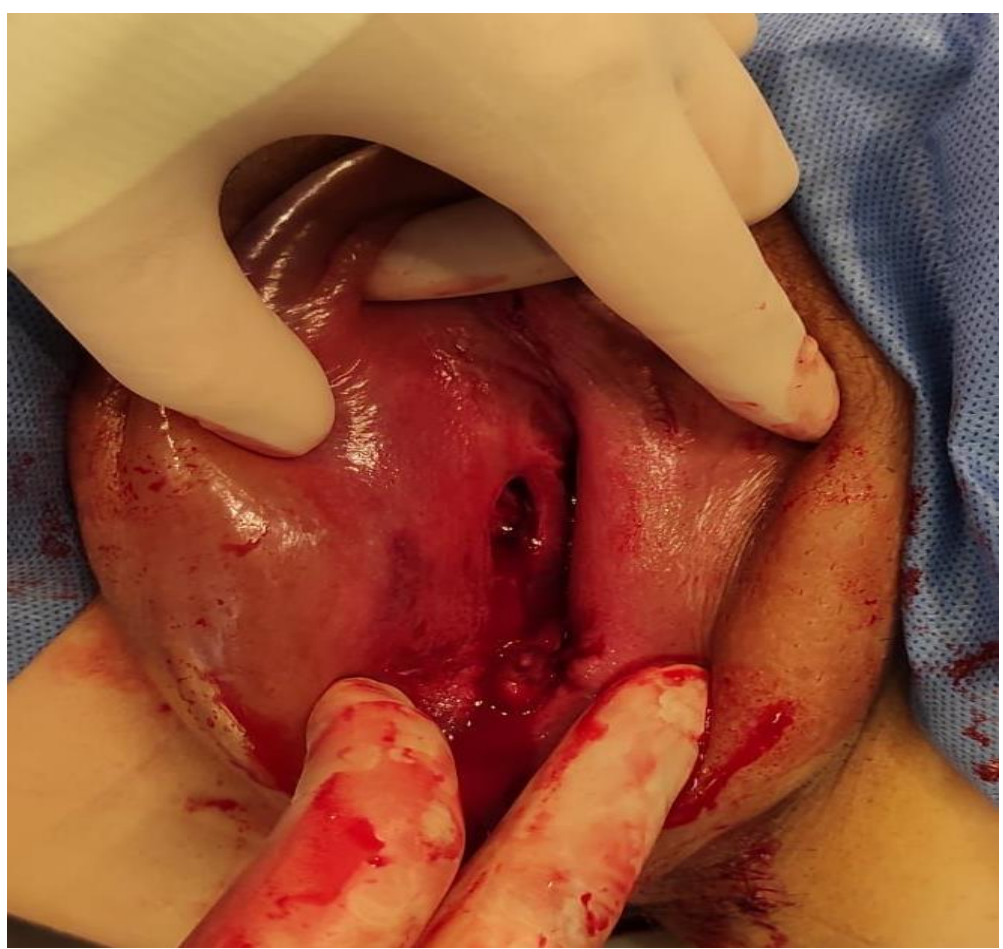

Figure 5. Incision, drainage, debridement and hemostasis in the lower third of the right labia majora.

During postoperative period and due to generalized and massive vulvar edema, blood testing was performed to rule out hypoproteinemia and / or hypoalbuminemia [5.82 grams of total proteins and 3.1 of albumin without other relevant alterations]. Intravenous analgesia and prophylactic antibiotic therapy were maintained for a week. As a treatment for the edema, she required: local applications of Glucosmón®, two doses of intravenous furosemide and intramuscular methylprednisolone. She was discharged 7 days postpartum, without bladder catheterization, local ice on demand, and compressive 
underwear. In the 15-days-follow-up, the complete resolution of the edema was observed [FIGURES 6 and 7].

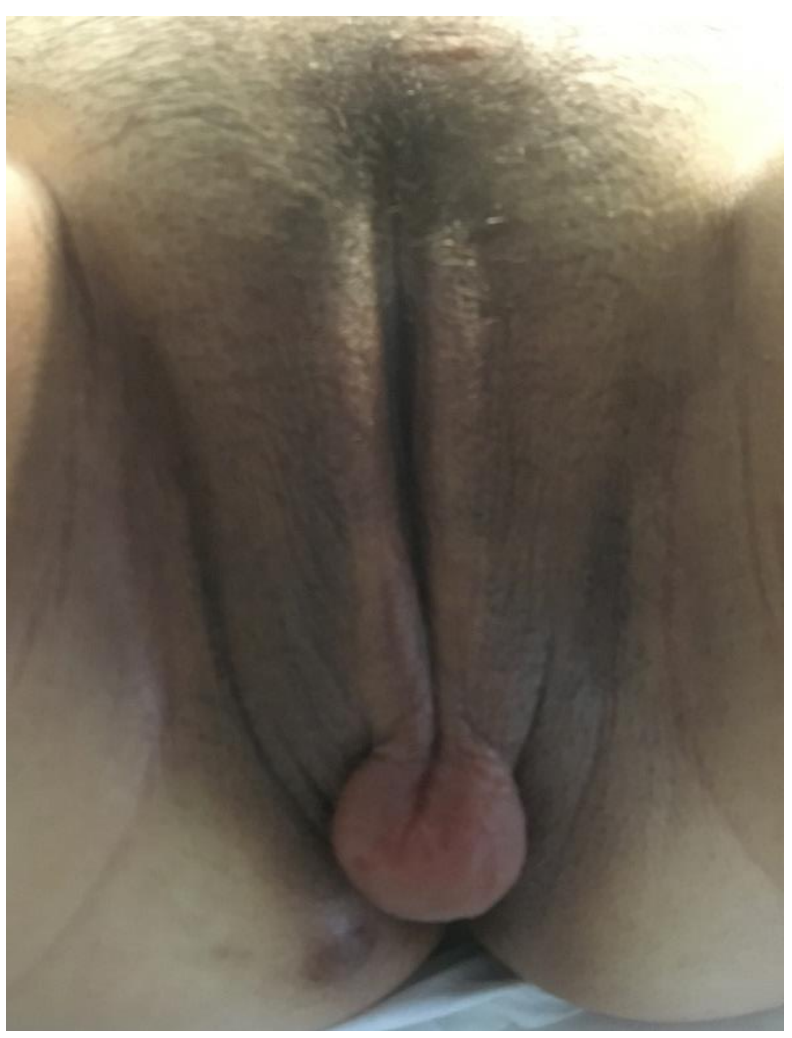

Figure 6. Evolution of vulvar edema 7 days postpartum.

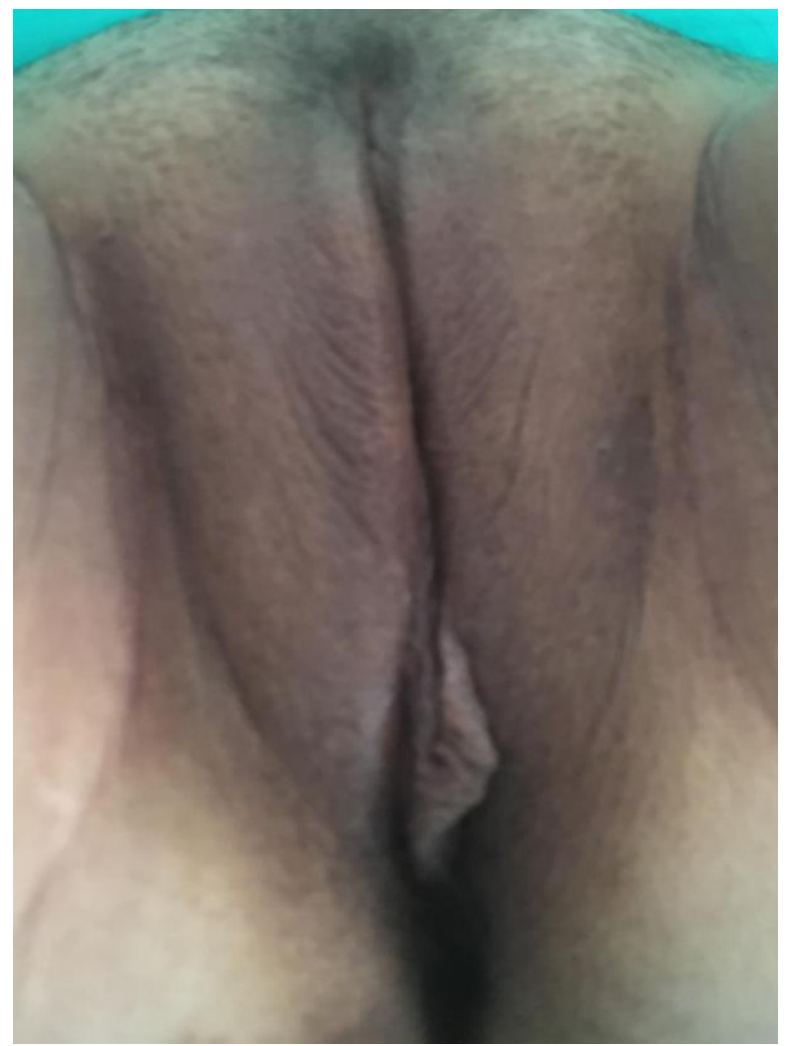




\section{Discussion}

The vulva or external genitalia comprise all visible structures from the symphysis pubis to the perineal body. Its main function is to protect the urinary meatus, vestibule, and vagina. It is composed of smooth muscle tissue and loose connective tissue, with abundant vascularization from the area of the pudendal artery, which is a branch of the internal iliac [10]. This rich blood supply makes it vulnerable to formation bruises after blunt trauma, particularly those that occur in a straddle position: bicycle accidents, athletics, traffic accidents or activities related with intercourse [2]. Risk factors for the development of non-obstetric vulvar hematomas include the absence of sexual intercourse, vaginal foreign objects or estrogen deprivation in menopause [11].

As we have previously mentioned, vulvar hematomas are much more frequent among the obstetric population due to the increased vascularization in the external and internal genitalia. In addition, there are certain risk factors that may predispose to the formation of puerperal hematomas: nulliparity, twin gestation or fetal macrosomia, preeclampsia, prolonged second stage, precipitous and instrumented delivery or vulvar varicose veins [5]. The patient in our second case had more than one risk factor.

A third much less frequent etiology is blood vessel spontaneous ruptures as a consequence of arteriovenous malformations, aneurysms or varicose veins. In non-obstetric hematomas, a detailed and directed history is essential for the etiological diagnosis [11].

The clinical signs that reveal the presence of a hematoma are: collection, lacerations, erythema, edema, ecchymosis, asymmetry of the lips and localized pain. Fluctuation of the lips, swelling and pain may indicate a developing bruise. If significant inflammation is seen without obvious bruising, translabial ultrasound can be useful to distinguish between edema and hematoma $[2,5]$.

The initial approach is indistinctive for all etiologies, with intravenous administration of plasma substitutes (Voluven 6\%®, solution of hydroxyethyl-starch) and the performance of a complete blood test, including hemogram and coagulation [5]. There are no data to support the use of prophylactic antibiotic therapy if surgical drainage is not to be performed.

The management of bruises is based on practices established throughout the experience since there are no illuminating clinical trials. There are three lines of treatment described: conservative management, surgical treatment, and selective arterial embolization. There is no agreement in the literature on whether conservative management is superior to surgical or vice versa [5].

The approach to treatment depends on: size, involvement of adjacent organs, hemodynamic involvement, and the existence of fractures or lacerations that need to be repaired [11].

Conservative management is recommended for small hematomas, under the premise that the inflammation of the soft tissues itself will block the origin of the bleeding with subsequent spontaneous resolution. In these cases, they are treated with local ice, limited physical activity, and analgesics in an ascending pattern for pain control [2, 5]. However, conservative management is associated with longer hospital stays and increased use of antibiotics and blood transfusions [12].

Surgical drainage is recommended for large hematomas or if the hematocrit drops steadily, which can lead to hemodynamic instability or tissue necrosis. Often, due to their size, they interfere with urination, so it is necessary to place a Foley catheter under sedation.
If surgical drainage is chosen, large incisions are recommended, allowing visualization of the bleeding vessels in order to be able to ligate them. The re-approximation of the edges of the skin has to be done with loose points of absorbable sutures, and the placement of a drain is useful. In the most complex cases, hemostatic agents can be used, and in very rare cases arterial embolization is necessary $[2,5]$, which is a more expensive procedure and depends largely on the availability in each center, the experience and skill of the radiologist who performs it [3].

Complementary postsurgical treatment includes sitz baths, rest, and nonsteroidal anti-inflammatory drugs $[2,5]$, as well as the management of associated edema. Any inflammatory or obstructive process can produce edema [7], including pregnancy. 8 out of 10 pregnant women have clinical edema at some point during pregnancy; physiologically the amount of body water increases from 6 to 8 liters [13]. This leads to a mild relative hypoproteinemia. Among the risk factors for developing obstetric vulvar edema; we found prolonged labor, pre-eclampsia, treatment with tocolytics [8], diabetes [14], ovarian hyperstimulation, severe hypoproteinemia and anemia as preexistent conditions [9].

Since most edema resolves spontaneously after delivery, its management is purely symptomatic through conservative measures such as local ice application and compression [8]. In moderate cases, the use of diuretics may be necessary. In the most severe ones, it is mandatory to rule out an underlying etiology other than the hematoma that is aggravating the process. In order to clarify, it is necessary to assess blood albumin concentrations (and correct severe hypoalbuminemia if it exists) and evaluate kidney and liver function [7]. In cases in which obstructive pathology of venous or lymphatic return is suspected, an imaging test may be considered to rule out tumor formation in the pelvis, or vascular thrombosis phenomena [9]. Treatment in these cases should be aimed at solving the underlying cause [7]. Only a few authors advocate mechanical drainage of edema in highly selected cases in which medical treatment fails [9].

In our patients, we chose surgical approach to the hematoma in both cases; given the uncontrolled pain, the extension and temporal progression of it and the compromise of the urinary tract. The patient in our first case required reoperation due to clinical worsening. We consider that it is possible that no active bleeding was observed in the first surgical procedure, perhaps due to an insufficient incision and plugged by the clots originated with the initial bleeding. When decompressing the surgical field, we understand venous bleeding restarted with new expansion of the vulvar hematoma. This highlights the importance of wide incisions that allow correct visualization and repair of possible arterial or venous injuries, as well as the closure of all the dissected spaces.

The particularity of the patient in our second case was the significant generalized edema that she already presented before delivery. Her condition worsened in the vulvar area after delivery, making it difficult to diagnose the hematoma of the right lip and delaying surgical drainage. She had several risk factors for the development of vulvar edema: she had a prolonged delivery, diabetes, and mild hypoproteinemia. Postoperatively, the severe edema made it necessary to associate conservative treatments in order to reduce it: furosemide, methylprednisolone and Glucosmón $®$. As discussed earlier, edema is a complication frequently associated with hematomas, which we must not lose sight of as it can delay the diagnosis of the hematoma and be even more disabling than the hematoma itself.

None of our patients presented infectious complications despite the vulva being a susceptible area; this fact could be explained by prompt removal of the urinary catheter, the use of antibiotic prophylaxis, and early 
mobilization. Both patients were discharged after a week, with significant clinical improvement and no sequelae, with a shorter mean stay than if conservative management had been chosen, although these cases did not meet the criteria for this.

\section{Conclusions}

- Although hematomas from traumatic causes are less frequent than postpartum ones, they can be very menacing as they can be associated with arterial bleeding that leads to hemodynamic instability.

- The use of intravenous analgesia or anesthetics is essential for pain management, since it is a densely innervated area.

- The approach can be conservative or surgical depending on the size, the hemodynamic compromise and adjacent organ involvement, and the presence of fractures or lacerations.

- Smaller bruises can be treated conservatively, reserving surgical management for more extensive bruises.

- Surgical management reduces hospital stay and the risk of infectiontion.

\section{ETHICAL APPROVAL}

Before obtaining the images of both clinical cases, their verbal consent was obtained, after informing the patients that their clinical processes could be the object of study and publication.

\section{References:}

1. Clínica Universidad de Navarra. Diccionario médico. Disponible en

2. Laufer MR, Makai G. (2021) Evaluation and management of female lower genital tract trauma. En: Mann WJ, Chakrabarti A, ed. UpToDate. Waltham, Mass.: UpToDate

3. Mangwi AA, Ebasone PV, Aroke D, Ngek LT, Nji AS. (2019) Non-obstetric vulva haematomas in a low resource setting: two case reports. Pan Afr Med J; 33:314.

4. Villella J, Garry D, Levine G, Glanz S, Figueroa R, Maulik D.(2001) Postpartum angiographic embolization for vulvovaginal hematoma. A report of two cases. J Reprod Med. 46(1):65-7.

5. Roman AS.(2021) Management of hematomas incurred as a result of obstetrical delivery. En: Berghella V, Eckler K, ed. UpToDate. Waltham, Mass.: UpToDate.

6. Hubb AJ, Orr KL, Stockdale CK.(2015) Puerperal vulvar edema and hematoma complicated by overuse of cold therapy: a report of two cases. J Low Genit Tract Dis; 19(2):e28-30.

7. Amankwah Y, Haefner H. Vulvar edema.( 2010) Dermatol Clin.; 28:765-777

8. Berens P. Overview of the postpartum period: Disorders and complications. En: Lockwood CJ, Barss VA, ed. UpToDate. Waltham, Mass.: UpToDate, 2021.

9. Martí-Gamboa S, Savirón R, Campillos-Maza JM.( 2014) Massive gestational vulvar edema. A case report and review of literature. Ginecol Obstet Mex.; 82:634-640.

10. Baggish MS. Anatomía vulvar y perineal. En: Baggish M, editor.( 2014) Atlas de anatomía de la pelvis y cirugía ginecológica. $4^{\circ}$ ed. Ciudad Autónoma de Buenos Aires: Médica Panamericana;. p. 823-840.

11. Lapresa MV, Hernández E, Bustillo S, Doyague MJ.(2019) Nonobstetric traumatic vulvar hematoma: Conservative or surgical approach? A case report. Case Rep Womens Health.; 22:e00109.

12. Benrubi G, Neuman C, Nuss RC, Thompson RJ.(1987) Vulvar and vaginal hematomas: a retrospective study of conservatuve versus operative management. South Med J.; 80(8):991-994.

13. Davison JM.(1987) Edema in pregnancy. Kidney Int Suppl.;59:S90-S96.
14. Mulisya O, Mastaki M, Gertrude T, Tasi K, Mathe JK.( 2018) Spontaneous Massive Vulvar Edema in Pregnancy: a case report. Case Reports in Obstetrics and Gynecology. 